\title{
An Ingenious Plasma Simulation Model for Large-Scale System with Self-Consistent Electron Dynamics
}

\author{
Tomonori TAKIZUKA, Kenzo IBANO and Masatoshi YAGI ${ }^{1)}$ \\ Graduate School of Engineering, Osaka University, Suita 565-0871, Japan \\ ${ }^{1)}$ National Institutes for Quantum and Radiological Science and Technology, Rokkasho 039-3212, Japan
}

(Received 12 April 2018 / Accepted 8 June 2018)

\begin{abstract}
An ingenious model for large-scale plasma simulations is proposed. An artificial permittivity $\varepsilon_{*}$ much grater than $\varepsilon_{0}$ (permittivity of free space) is introduced to the Poisson equation, $\nabla \cdot\left(\varepsilon_{*} \nabla \phi\right)=-\rho(\phi$ is electrostatic potential and $\rho$ is charge density), and the Debye length is artificially elongated. A large-scale system with a reasonable number of spatial grids can be treated stably even for including the self-consistent electron dynamics. A dielectric tensor is adopted for the three-dimensional global simulation of tokamak plasmas in the cylindrical coordinates $(R, \theta, Z)$. A diagonal element $\varepsilon_{\theta \theta}$ for toroidal axis $\theta$ is set much larger than those for the poloidal plane, $\varepsilon_{R R}=\varepsilon_{Z Z}=\varepsilon_{*}$. Number of toroidal meshes $N_{\theta}$ can be reduced enough smaller than that of poloidal-plane meshes, $N_{R}$ and $N_{Z}$, in comparison with $N_{\theta}>N_{R}, N_{Z}$ for the usual case of isotropic $\varepsilon_{*}$.
\end{abstract}

(C) 2018 The Japan Society of Plasma Science and Nuclear Fusion Research

Keywords: simulation model, global plasma, electron dynamics, Poisson equation

DOI: $10.1585 /$ pfr. 13.1203088

Numerical simulation of plasma dynamics is a powerful tool to understand the underlying physics in experimental observations for various fields of plasma science, such as thermonuclear fusion, astrophysics, applied physics etc. It is also an indispensable means to predict the plasma natures in future experiments, such as fusion reactor etc. The global plasma simulation is especially important for a fusion plasma. Majorities of global plasma simulations have assumed the quasi-neutral condition, and have not directly solved electron dynamics. When the electron dynamics are self-consistently traced, the plasma frequency $\omega_{\mathrm{p}}=$ $\left(e^{2} n_{\mathrm{e}} / \varepsilon_{0} m_{\mathrm{e}}\right)^{1 / 2}$ and the Debye length $\lambda_{\mathrm{D}}=\left(\varepsilon_{0} T_{\mathrm{e}} / e^{2} n_{\mathrm{e}}\right)^{1 / 2}$ restrict the time step $\Delta t$ and spatial mesh size $\Delta$ to very small ones, respectively $\left(\varepsilon_{0}\right.$ : permittivity of free space, $e$ : elementary charge, $m_{\mathrm{e}}$ : electron mass, $n_{\mathrm{e}}$ : electron density, $T_{\mathrm{e}}$ : electron temperature). Resultantly computation cost becomes extremely expensive. The electron dynamics, however, play important roles in a global plasma especially for the case of interacting with peripheral walls. Therefore we propose here an ingenious model to reduce this expensive cost, by focusing the electrostatic Particle-inCell (PIC) simulation of tokamak plasmas including SOLdivertor regions.

In order to study SOL-divertor plasmas in a tokamak, a particle code PARASOL has been developed and fruitful physics results have been demonstrated [1,2]. Electron dynamics are self-consistently traced and the sheath in front of a material wall is actually simulated in PARASOL. Kinetic effects, e.g., the limited parallel heat flux in a rare-collision plasma compared to the collisional Spitzer-

author'se-mail: takizuka.tomonori@gmail.com
Harm heat flux, are accurately described as well. Onedimensional (1D) simulations of ITER SOL-divertor plasmas, for instance, were successfully performed, where the system length was $L \sim 30 \mathrm{~m}$ and number of grids was $N \sim 10^{4}$. The mesh size $\Delta \sim 3 \mathrm{~mm}$ was much larger than $\lambda_{\mathrm{D}} \sim 0.01 \mathrm{~mm}$ for $n_{\mathrm{e}} \sim 10^{20} / \mathrm{m}^{3}$ and $T_{\mathrm{e}} \sim 100 \mathrm{eV}[2,3]$. Recently 2D PARASOL simulations for a small tokamak like COMPASS were carried out, where $L_{R} \times L_{Z} \sim 0.6 \mathrm{~m}$ $\times 1 \mathrm{~m}, N_{R} \times N_{Z} \sim 600 \times 1000$, and $\Delta \sim 1 \mathrm{~mm}$ was also much larger than $\lambda_{\mathrm{D}} \sim 0.01 \mathrm{~mm}$ for $n_{\mathrm{e}} \sim 3 \times 10^{19} / \mathrm{m}^{3}$ and $T_{\mathrm{e}} \sim 70 \mathrm{eV}[4,5]$. If a conventional PIC simulation is executed under the condition $\Delta \gg \lambda_{\mathrm{D}}$, numerical instability called grid instability occurs and decent results are never obtained [6].

To avoid this instability even for the small number of grids in PARASOL simulations, the artificially elongated Debye length $\lambda_{\mathrm{D} *}$ has been adopted so that the condition $\Delta / \lambda_{\mathrm{D} *} \sim 1$ is satisfied [1,2]. The elongation of $\lambda_{\mathrm{D}}$ is simply made by replacing $\varepsilon_{0}$ with artificially enlarged one $\varepsilon_{*} \gg \varepsilon_{0}$ in the Poisson equation as

$$
\nabla \cdot\left(\varepsilon_{*} \nabla \phi\right)=-\rho,
$$

where $\phi$ is the electrostatic potential. The charge density $\rho$ is calculated from the finite-size particle positions of electrons and ions in the PIC simulation. In the above PARASOL simulations for 1D ITER and 2D COMPASS, the values of $\varepsilon_{*}$ were set $10^{4} \sim 10^{6}$ times larger than $\varepsilon_{0}$. It is noted that $\omega_{\mathrm{p} *}$ becomes small simultaneously, and $\Delta t \sim O\left(1 / \omega_{\mathrm{p} *}\right)$ can be chosen reasonably large in the simulation. This artificial setting is allowable because the characteristics of SOL-divertor plasmas under the quasineutral condition are similar to the core plasma [7] and are 


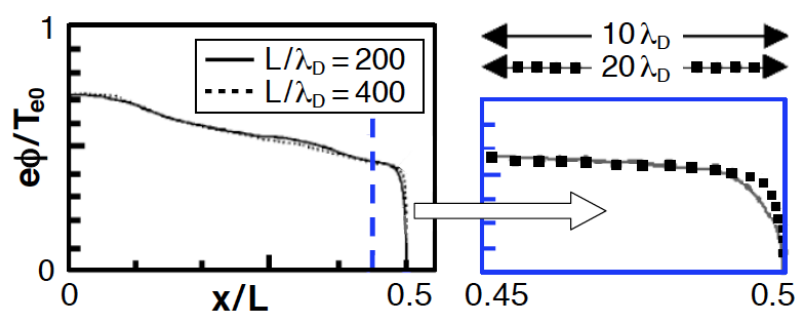

Fig. 1 Potential profiles for different $L / \lambda_{\mathrm{D}}$ values. The same profiles are established except in the sheath region.

governed mainly by collisionality and normalized ion Larmor radius but insensitive to the $L / \lambda_{\mathrm{D}}$ ratio as shown in Fig. 1. The difference is only seen in the sheath region in front of the material wall (right figure). It is worthy to note that this profile is a time-averaged one over a hundred $\Delta t$, and high-frequency oscillations are filtered out $[1,2]$.

Another high-frequency dynamics of electrons, gyro motion, is removed by adopting guiding-center equations in a strong magnetic field $\boldsymbol{B}$. The electric field $\boldsymbol{E}$ consists of self-consistent electrostatic field $\boldsymbol{E}_{\mathrm{s}}=-\nabla \phi$ and inductive field $\boldsymbol{E}_{\text {ind }}$ such as an applied toroidal electric field. Drift velocities of $E \times B$ drift and curvature- $\nabla B$ drift are taken into account, but the polarization drift is neglected. As for the ion motion, its gyration is fully followed and the polarization drift is exactly simulated as well. Coulomb collisions are very important to determine the plasma properties especially in edge plasmas. A binary collision model is very useful for the PIC simulation [8].

In SOL-divertor plasmas, particles and heats are lost to the divertor plates mainly along $\boldsymbol{B}$. The hot plasma is supplied at the central source region in a $1 \mathrm{D}$ system. For a 2D system, the hot plasma is diffused out from the confined core region to the SOL region through a Monte-Carlo random-walk diffusion model $[1,2]$. Thus the PARASOL has succeeded in the simulations of large-scale 1D and 2D systems for tokamak plasmas.

In order to study tokamak edge plasmas including the turbulence-driven transport nature, reliable 3D simulations are indispensable. Although the introduction of artificially elongated $\lambda_{\mathrm{D} *}$ enables us to handle a large-scale 2D tokamak system within a reasonable number of spatial grids, it is still difficult to try a 3D PIC simulation. Because the toroidal length $L_{\theta}=2 \pi R$ is larger than horizontal and vertical lengths of the vessel $L_{R}$ and $L_{Z}$, the number of grids along toroidal direction $N_{\theta}$ has to be larger than those on the poloidal plane, $N_{R}$ and $N_{Z}$, when every mesh size $\Delta=L / N$ is kept of the order of $\lambda_{\mathrm{D} *}$ for every direction. Here we use cylindrical coordinates $(R, \theta, Z)$. Considering $N_{R} \sim N_{Z} \sim 10^{3}$, particle number in a cell $\mathcal{N}_{\text {cell }} \sim 10^{2}$, total particle number $\mathcal{N}_{\text {tot }} \sim 10^{8}$ and time steps $K_{\mathrm{t}} \sim 10^{6}$ for a 2D PIC simulation of a tokamak plasma, we estimate the operations $\sim 10^{17}$, the efficiency $\sim 10 \%$, and the run time $\sim 10^{3} \mathrm{~s}$ using a 1 PFLOPS supercomputer. Since $\mathcal{N}_{\text {tot }}$ for a $3 \mathrm{D}$ case becomes $\sim N_{\theta}$ times larger than that for a $2 \mathrm{D}$ case, the computation time of 1 hour for a $2 \mathrm{D}$ sim- ulation increases more than 1 month for a 3D simulation with $N_{\theta}>10^{3}$. Therefore 3D PIC simulations with $N_{\theta}$ of the order of $10^{2}$ are desirable, which can be executed repetitively every week. The poloidal-plane mesh numbers $N_{R} \sim N_{z} \sim 10^{3}$ for the above example are enough to treat the edge-plasma turbulence with the poloidal mode number $m$ up to 100. Assuming the safety factor $q$ in the tokamak peripheral is greater than 3 , the toroidal mode number $n$ required to treat the edge-plasma turbulence, $n \sim m / q$, is about 30. The corresponding toroidal mesh number $N_{\theta}$ could be $\sim 200$.

Now we propose an ingenious model to reduce $N_{\theta}$ without bringing about the grid instability due to $\Delta_{\theta}>\lambda_{\mathrm{D} *}$. In this new model, the permittivity $\varepsilon_{*}$ in Eq. (1) is further modified to a dielectric tensor $\ddot{\varepsilon}$, whose diagonal elements are $\varepsilon_{R R}=\varepsilon_{Z Z}=\varepsilon_{*}$ and $\varepsilon_{\theta \theta}=\alpha^{2} \varepsilon_{*}$ with $\alpha \gg 1$. The size $\Delta_{\theta}$ can be set $\alpha$ times larger than $\Delta_{R}$ and $\Delta_{Z}$. The change of $\varepsilon$ value (or $\lambda_{\mathrm{D}}$ size) does not affect the $\phi$ profile in a quasi-neutral plasma as above mentioned, while it alters the amount of charge separation $\rho$ keeping the $\phi$ profile. Thus it becomes possible to carry out 3D simulations with reasonably small number of toroidal meshes $N_{\theta}$. Basing on the above example for $N_{R} \sim N_{Z} \sim 10^{3}$, a 3D simulation with $N_{\theta} \sim 200(\alpha \sim 20)$ and $K_{\mathrm{t}} \sim 10^{6}$ will be executed within a week. Here the turbulence modes can be treated up to $n \sim 30$ and $m \sim 150$. Using a certain time slice as an initial condition, a subsequent simulation with larger $N_{\theta}$ (setting $1<\alpha<10$ ) and shorter $K_{\mathrm{t}} \sim 2 \times 10^{8} / N_{\theta}$ is executed to verify the result of anisotropic $\ddot{\varepsilon}$ for small $N_{\theta}$ simulation.

If a 3D PIC code based on the above ingenious model is developed, it will be useful to study various unresolved issues for tokamak edge plasmas, such as "SOL heat-flux width", "blob transport", "far SOL", "ELM filament transport", "density limit", "L-H transition", and so on.

Finally we note that the present modeling is applicable to the two-fluid simulation in which electron dynamics and Poisson equation are self-consistently solved as the same as in the PIC simulation.

We acknowledge Mr. M. Hosokawa of ITER organization for exploratory discussion on the large-scale 3D PARASOL simulation towards ITER.

[1] T. Takizuka, Plasma Sci. Technol. 13, 316 (2011).

[2] T. Takizuka, Plasma Phys. Control. Fusion 59, 034008 (2017).

[3] M. Hosokawa, A. Loarte, G.T.A. Huijsmans, T. Takizuka and N. Hayashi, Plasma Fusion Res. 11, 1403104 (2016).

[4] M. Hosokawa et al., 16th Int. Workshop on Plasma Edge Theory in Fusion Devices (Marseille, France, 2017).

[5] T. Takizuka, M. Hosokawa, A. Loarte and N. Hayashi, Proc. 15th BPSI meeting (Kasuga, 2017), http://www.riam. kyushu-u.ac.jp/sosei/bpsi/2017/BPSI2017_proc.pdf

[6] R.W. Hockney and J.W. Eastwood, Computer Simulation Using Particles (New York, McGraw-Hill, 1981).

[7] J.W. Connor and J.B. Taylor, Nucl. Fusion 17, 1047 (1977).

[8] T. Takizuka and H. Abe, J. Comput. Phys. 25, 205 (1977). 